\title{
1 Switching of OAS1 splicing isoforms mitigates SARS-CoV-2 infection
}

3 Kei Iida ${ }^{1 * \#}$, Masahiko Ajiro ${ }^{2,3 *}$, Yukiko Muramoto ${ }^{4,5}$, Toru Takenaga ${ }^{4,5}$, Masatsugu Denawa ${ }^{1}$, Ryo

4 Kurosawa $^{3}$, Takeshi Noda ${ }^{4,5}$, Masatoshi Hagiwara ${ }^{3 \#}$

$5{ }^{1}$ Medical Research Support Center, Graduate School of Medicine, Kyoto University, Yoshida-Konoe-cho,

6 Sakyo-ku, Kyoto 606-8501, Japan

$7 \quad{ }^{2}$ Department of Drug Discovery Medicine, Graduate School of Medicine, Kyoto University, Yoshida-

8 Konoe-cho, Sakyo-ku, Kyoto 606-8501, Japan

$9{ }^{3}$ Department of Anatomy and Developmental Biology, Graduate School of Medicine, Kyoto University,

10 Yoshida-Konoe-cho, Sakyo-ku, Kyoto 606-8501, Japan

$11{ }^{4}$ Laboratory of Ultrastructural Virology, Institute for Frontier Life and Medical Sciences, Kyoto University,

12 Kyoto 606-8507, Japan.

$13{ }^{5}$ CREST, Japan Science and Technology Agency, 4-1-8 Honcho, Kawaguchi, Saitama 332-0012, Japan

14

$15 *$ These authors share first authorship.

16 \# These authors share last authorship.

17

18 Corresponding authors:

19 Kei Iida, PhD

20 Research Assistant Professor

21 Medical Research Support Center, Graduate School of Medicine,

22 Kyoto University Yoshida-Konoe-cho, Sakyo-ku, Kyoto 606-8501, Japan

23 E-mail: iida.kei.3r@kyoto-u.ac.jp

25 Masatoshi Hagiwara, MD, PhD

26 Professor

27 Department of Anatomy and Developmental Biology Graduate School of Medicine,

28 Kyoto University Yoshida-Konoe-cho, Sakyo-ku, Kyoto 606-8501, Japan

29 E-mail: hagiwara.masatoshi.8c@kyoto-u.ac.jp 


\section{$31 \quad$ Abstract}

32 (Background) The rapidly accumulating disease susceptibility information collected from coronavirus

33 disease (COVID-19) patient genomes must be urgently utilized to develop therapeutic interventions

34 for SARS-CoV-2 infection. Chromosome 12q24.13, which encodes the 2'-5'-oligoadenylate synthetase

35 (OAS) family of proteins that sense viral genomic RNAs and trigger an antiviral response, is

36 identified as one of the genomic regions that contains SNPs associated with COVID-19 severity. A

37 high-risk SNP identified at the splice acceptor site of $O A S 1$ exon 6 is known to change the proportions

38 of the various splicing isoforms and the activity of the enzyme.

39 (Methods) We employed in-silico motif search and RNA pull-down assay to define a factor

40 responsible for the $O A S 1$ splicing. Next, we rationally selected a candidate for slicing modulator to

41 modulate this splicing.

42 (Results) We found that inhibition of CDC-like kinase with a small chemical compound induces

43 switching of $O A S 1$ splice isoforms in human lung cells. In this condition, increased resistance to

44 SARS-CoV-2 infection, enhanced RNA degradation, and transcriptional activation of interferon $\beta 1$,

45 were also observed.

46 (Conclusions) The results indicate the possibility of using chemical splicing modifiers aided by

47 genome-based precision medicine to boost the innate immune response against SARS-CoV-2

48 infection.

49

50 Keywords: SARS-CoV-2, OAS-1, splice forms

51

\section{$52 \quad$ Funding}

53 This study was supported by grants 15H05721 (to M.H., K.I., and M.A.), 19K07367 (to M.A.) and

5420 K07310 (to K.I.) from the Japan Society for the Promotion of Science, the Kansai Economic

55 Federation (KANKEIREN) (to M.H.), Research Program on Emerging and Re-emerging Infectious

56 Disease grants JP20fk0108270 (to M.H. and T.N.) and JP20ek0109327 (to M.H., and M.A.) from 
57 AMED, the JST Core Research for Evolutional Science and Technology grant JPMJCR20HA (to

58 T.N.), and the Grant for Joint Research Project of the Institute of Medical Science, University of

59 Tokyo, and the Joint Usage/Research Center program of Institute for Frontier Life and Medical

60 Sciences Kyoto University (to T.N.).

\section{Introduction}

63 As of July 2021, more than 180 million people have been infected with SARS-CoV-2 and more than

644.0 million people have died of COVID-19 (https://covid19.who.int). Despite extensive efforts

65 towards prophylactic vaccination against SARS-CoV-2, the incidence of COVID-19 continues to

66 increase, with rapid emergence of mutant strains (https://www.who.int/). To understand SARS-CoV-2

67 pathogenesis and develop novel therapeutic approaches, it is essential to analyze genetic factors

68 responsible for susceptibility and severity. A previous genome-wide association study (GWAS) in

69 over 2,000 COVID-19 patients uncovered several susceptibility-associated genes, including LZTFL1,

70 CCHCR1, OAS1, OAS2, OAS3, DPP9, TYK2, and IFNAR2 (Pairo-Castineira et al., 2021). The

71 COVID-19 host genetics initiative also gathered genetic information for more than 30,000 COVID-19

72 patients, including over 5,000 patients with very severe respiratory symptoms, and showed the similar

73 results (The COVID-19 Host Genetics Initiative, 2021). It revealed 131 SNPs associated with severe

74 respiratory conditions $\left(\mathrm{p}<5 \times 10^{-8}\right)$ in the gene cluster of $O A S 1, O A S 2$, and $O A S 3$ (Fig. 1a). They

75 consist of 5 SNPs within the OAS1 locus, 72 SNPs within intergenic regions across OAS1, OAS2, and

76 OAS3, 46 SNPs within the $O A S 3$ locus, and 8 SNPs within the OAS2 locus. Importance of the $O A S$

77 family of genes was further indicated in a transcriptome study in an in vitro model of SARS-CoV-2

78 infection (Blanco-Melo et al., 2020), in which normal human bronchial epithelial (NHBE) cells

79 exhibited an increased expression of $O A S 1, O A S 2$, and $O A S 3$ (2.3, 2.1, and 2.2-fold increase,

80 respectively). Of the aforementioned SNPs, this genomic region includes SNPs associated with

81 hospitalized COVID-19 patients (32 SNPs) and all COVID-19 patients (125 SNPs) with low p-values

$82\left(\mathrm{p}<5 \times 10^{-8}\right)($ Fig. 1b, 1c), which are concentrated around the terminal exon of OAS1 and intron 2 of 
83 OAS3. Amongst them, the SNP with the lowest p-value was the G>A SNP rs10774671, located at the

84 last base of intron 5 (Table 1), and its association with infectious viruses, including SARS coronavirus

85 and Dengue virus, has been reported (Hamano et al., 2005; He et al., 2006; Lin et al., 2009).

\section{$88 \quad$ Materials and Methods}

89 Analysis of GWAS data

90 GWAS results were obtained from the COVID-19 Host Genetics Initiative data. The following terms

91 were used: “A2_ALL_leave_23andme” for GWAS of confirmed COVID patients with very severe

92 respiratory conditions vs. population, "B2_ALL_leave_23andme" for GWAS of hospitalized COVID

93 patients vs. population, and "C2_ALL_leave_23andme" for GWAS of all COVID patients vs.

94 population. Data version round 5 was used for all the data obtained in this study (January 18, 2021).

95 The sample size of 5582, 12888, and 36590 individuals were chosen for evaluation of GWAS datasets

96 of severe respiratory symptoms, hospitalization, and COVID-19 susceptibility, respectively, obtained

97 from COVID-19 host genome initiative database (The COVID-19 Host Genetics Initiative, 2021).

98 This sample number was determined based on number of applicable data without exclusion, and a

99 sample-size estimation was not conducted prior to the analysis.

Cell lines

102 Daudi cells, derived from Burkitt's lymphoma, were obtained from the cell bank of the National

103 Institutes of Biomedical Innovation, Health and Nutrition (NIBIOHN, Osaka, Japan) and maintained

104 in RPMI 1640 medium (Nacalai Tesque, Kyoto, Japan) supplemented with 20\% fetal bovine serum,

$105100 \mathrm{U} / \mathrm{mL}$ penicillin, and $100 \mu \mathrm{g} / \mathrm{mL}$ streptomycin. Calu-3 cells, derived from lung adenocarcinoma,

106 were obtained from American Type Culture Collection (ATCC, Manassas, VA, USA), and cultured in

107 Dulbecco's modified Eagle's medium (DMEM) (Nacalai Tesque) supplemented with $10 \%$ fetal

108 bovine serum, $100 \mathrm{U} / \mathrm{mL}$ penicillin, and $100 \mu \mathrm{g} / \mathrm{mL}$ streptomycin. All cells were maintained in an 
109 incubator at $37{ }^{\circ} \mathrm{C}$ with $5 \% \mathrm{CO}_{2}$, and mycoplasma was confirmed negative in routine polymerase

110 chain reaction tests. VeroE6/TMPRSS2 cells were obtained from JCRB Cell Bank, and cultured in

111 Dulbecco's modified Eagle's medium (DMEM) (Sigma-Aldrich) supplemented with $10 \%$ fetal

112 bovine serum, $100 \mathrm{U} / \mathrm{mL}$ penicillin, and $100 \mu \mathrm{g} / \mathrm{mL}$ streptomycin. The cells were maintained in an

113 incubator at $37^{\circ} \mathrm{C}$ with $5 \% \mathrm{CO} 2$.

RNA pull-down assay and western blot

116 Calu-3 whole cell lysates were prepared using a lysis buffer containing $10 \mathrm{mM}$ Tris- $\mathrm{HCl}(\mathrm{pH} 7.4), 150$

$117 \mathrm{mM} \mathrm{NaCl}, 1 \mathrm{mM}$ ethylenediaminetetraacetic acid, $1 \%$ Triton X-100, 0.1\% sodium dodecyl sulphate,

$118 \quad 0.25 \%$ sodium deoxycholate, and $10 \%$ glycerol with protease inhibitors (Nacalai Tesque) and

119 phosphatase inhibitors (Sigma-Aldrich, Munich, Germany), followed by sonication, treatment with

120 DNase I (Promega, Madison, WI, USA) at $37{ }^{\circ} \mathrm{C}$ for $5 \mathrm{~min}$, and centrifugation $\left(24,000 \times \mathrm{g}\right.$ at $4{ }^{\circ} \mathrm{C}$ for

$12115 \mathrm{~min})$. The supernatant was used as the soluble fraction for the RNA-pull down assay.

122 For the RNA pull-down assay, 5'-biotin, 3'-dTdT-attached RNA, designed for the sequence adjacent

123 to the OAS1 exon 5 splice donor (5'-CUGCUGGUGAGACCUCCUGCUUCC-3' (oAM685), was

124 incubated with NeutrAvidin beads (Thermo Fisher Scientific, Waltham, MA, USA) for $2 \mathrm{~h}$ at $4{ }^{\circ} \mathrm{C}$ (no

125 bait RNA was used for the negative control), followed by wash with 1X TBS thrice. RNA-bound

126 NeutrAvidin beads were then incubated with the Calu-3 cell lysate in the presence of $1 \%$ DMSO or 10

$127 \mu \mathrm{M}$ CaNDY for $16 \mathrm{~h}$ with rotation at $4{ }^{\circ} \mathrm{C}$. This was followed by washing thrice with tris-buffered

128 saline and elution with Laemmli buffer. Three technical replicates for RNA pull-down assay were

129 independently conducted, three times from a stocked cell lysate. Eluted proteins were then analyzed

130 by western blotting with anti-U1-70k mouse monoclonal antibody (9C4.1) (05-1588, Merk Millipore,

131 Burlington, MA, USA) at a dilution of 1:500 for the detection of U1-70k, anti-SR protein (1H4G7)

132 mouse monoclonal antibody (33-9400, Thermo Fisher Scientific, Waltham, MA, USA) at a dilution of

133 1:200 for phosphorylated SRSF6, and anti- $\beta$-actin (ACTB) mouse monoclonal antibody (Ac-15) (sc-

134 69879, Santa Cruz Biotechnology, Dallas, TX, USA) at a dilution of 1:4,000 for ACTB. 
135 Chemiluminescent signals were detected using a ChemiDoc MP Imaging System (Bio-Rad, Hercules,

\section{CA, USA).}

139 RNAs were extracted using RNeasy Mini kit (QIAGEN, Hilden, Germany) from Calu-3 cells, treated

140 with $10 \mu \mathrm{M}$ CaNDY or $0.1 \%$ DMSO for $18 \mathrm{~h}$, and applied for RNA-Seq analysis. Three technical

141 replicates for RNA-Seq, where each experiment was independently conducted three times from a

142 stocked total RNA. RNA-seq reads were mapped to the human genome sequences (GRCh38) using

143 STAR (ver. 2.7.1a, https://github.com/alexdobin/STAR) with ENCODE options, using the Ensembl

144 genome annotation (ver. 102). Raw reads were counted with bam files, and TPM values were

145 calculated using RSEM v1.2.31 (https://github.com/deweylab/RSEM). Differentially expressed genes

146 were identified using the method described above. Differential alternative splicing (DAS) events were

147 analyzed with the method previously described (Sakuma et al., 2015) using the rMATS program

148 (http://rnaseq-mats.sourceforge.net/rmats4.1.1/). DAS was defined by the following criteria: FDR <

1490.01 , read counts $\geq 15$, and delta Percent Spliced-In (PSI) $\geq 0.05$. We compared the DAS events with

150 the gene annotation information, then classified the event types into events on the exons constituting

151 the productive mRNAs, events on additional exons/regions of the productive mRNAs, and others.

152 For characterizing gene set and transcriptome profiles, we used the Metascape website

153 (https://metascape.org/) and Gene Set Enrichment Analysis (GSEA, https://www.gsea-

154 msigdb.org/gsea/). The same samples were also used for RT-PCR.

SARS-CoV-2 infection

157 Calu-3 cells were pre-treated with $10 \mu \mathrm{M}$ CaNDY or $0.1 \%$ DMSO for $24 \mathrm{~h}$, infected with SARS-CoV-

1582 (SARS-CoV-2/Hu/DP/Kng/19-027) at a multiplicity of infection of 0.01 , and maintained in the

159 presence of $10 \mu \mathrm{M}$ CaNDY or $0.1 \%$ DMSO. RNA samples were collected from the cells $24 \mathrm{~h}$ post-

160 infection (pi), and the virus titers were determined by the $50 \%$ tissue culture infectious dose $\left(\mathrm{TCID}_{50}\right)$ 
using VeroE6/TMPRSS2 cells at $48 \mathrm{~h}$ pi. Titer assay was conducted in six biological replicates for independent cell cultures.

Analysis of RNA degradation

165 RNA samples were diluted to $200 \mathrm{ng} / \mu \mathrm{L}$. The quality of the diluted RNA samples was evaluated using

166 the Agilent RNA 6000 Nano Kit and the Agilent 2100 Bioanalyzer. For the infected cells, three

167 biological replicates with independent cell cultures were prepared for DMSO and CaNDY treatments, respectively, with the method described above. Total RNA from transcriptome analysis represented uninfected control for this study. The gel-like image of the 2100 Bioanalyzer result was visualized using the 2100 Expert Software (ver. B.02.11, Agilent Technologies) in pseudo colors with default

171 settings. For detailed analyses, the migration time and fluorescence unit data were extracted in CSV

172 format as aligned with the sample data. Next, the fluorescence unit values were normalized to an RNA

173 concentration of $300 \mathrm{ng} / \mu \mathrm{L}$. We selected the data located between $43.3 \mathrm{~s}$ and $48.0 \mathrm{~s}$ of the migration

174 time as cleaved RNA products and those between $49.9 \mathrm{~s}$ and $51.4 \mathrm{~s}$ as the $28 \mathrm{~S}$ rRNA. The sums of the

175 fluorescence unit values were used for further analysis. For drawing the bar plot, the values were

176 scaled to the mean values of DMSO samples.

$178 \quad R T-P C R$

179 Total RNA extracted from cultured cells was used for reverse transcription using the PrimeScript

180 RTase (Takara Bio, Shiga, Japan) with random hexamers, and the products were then amplified with

181 ExTaq DNA polymerase (Takara Bio) with target-specific primer sets. Primers used in RT-PCR are

182 listed in Table S1. Detection of RT-PCR products was conducted using the ChemiDoc MP Imaging

183 System (Bio-Rad), with subsequent analysis by Image Lab software (Bio-Rad). RT-PCR was

184 conducted in three technical replicates for a total RNA sample.

186 Modelling of the viral infection 
187 The statistical simulation of SARS-CoV-2 infections was performed with the SIR (Susceptible,

188 Infectious, or Recovered) model (Harko et al., 2014) using the "deSolve" package (Soetaert et al.,

189 2010) in the R environment. We assigned the initial number of susceptible persons as 9999 and

190 infectious person as 1. Beta, a parameter for infection rate per day, per person, was set as 0.5 or 0.28

191 (i.e., $0.5 \times 0.56$ ), and Gamma, a parameter for the removal or the recovery rate per day, per person,

192 was set as 0.1, according to a report on the spread of SARS-CoV-2 in April 2020, in the USA (Adam,

193 2020).

Data Availability

196 The datasets generated during and/or analyzed during the current study are available from the

197 corresponding author on reasonable request. The original RNA-seq data were deposited at the Gene

198 Expression Omnibus (GEO) of National Center for Biotechnology Information (NCBI) with the

199 accession ID GSE174398.

\section{Results}

202 OAS1 splice variant regulation by SRSF6

203 The rs $10774671 \mathrm{~A} / \mathrm{G}$ SNP is known to control the production of OAS1 splice variants. The G allele of

204 rs10774671 (G-allele) creates the AG-dinucleotide that is essential for the recognition of the exon 6

205 splice acceptor site for $p 46$ variant production. The A allele of rs 10774671 (A-allele) leads to the

206 alternative splicing of $O A S 1$ pre-mRNA to produce $p 42, p 48, p 44 a$, and $p 44 b$ variants (Noguchi et al.,

207 2013) (Fig. 2a). OAS1 protein senses the double-stranded RNA structure, including RNA duplication

208 intermediates of SARS-CoV-2 (Sadler and Williams, 2008; Schlee and Hartmann, 2016), to

209 synthesize 2'-5'-oligoadenylates, which in turn trigger the activation of latent ribonuclease L (RNase

210 L) for viral RNA degradation (Sadler and Williams, 2008; Schlee and Hartmann, 2016). Previous

211 studies revealed that the catalytic activity of OAS1 varies with its splice variants; p46, the major

212 isoform of OAS1 produced in the presence of the G-allele, presents optimal activity, while p42, the 
213 major isoform produced in the presence of the A-allele, shows poor activity (Carey et al., 2019; Di et

214 al., 2020).

215 In A-allele individuals, disruption of the exon 6 splice acceptor site induces $O A S 1$ alternative splicing,

216 yielding $p 44 a, p 44 b$, and $p 48$ through activation of acceptor sites downstream of the exon 5 , and $p 42$

217 as a consequence of the skipped recognition of exon 5 donor site (Fig. 2a). Assuming that the splice-

218 shift from the inactive $p 42$ to other variants may improve the immune-response that is weakened due

219 to the presence of $O A S 1$ A-allele, we first looked into the mechanism by which $p 42$ variant is

220 dominantly produced. In searching for splice motifs surrounding the OAS1 exon 5 donor sites with the

221 ESEfinder tool (Cartegni et al., 2003), we noticed a binding motif for serine/arginine-rich splicing

222 factor 6 (SRSF6) (5'-UGCUUC-3') immediately downstream to the exon 5 donor site (Fig. 2b). We

223 then speculated that SRSF6 interaction with this site could prevent U1snRNP from binding to the exon

2245 donor site. To test this hypothesis, we applied a pan-CDC-like kinase (CLK) inhibitor, CaNDY, to

225 dissociate SRSF6 from RNA by preventing the kinase activity of CLK to phosphorylate RS domain at

226 the carboxyl-terminal of SRSF6 (Shibata et al., 2020). Following an RNA pull-down assay, we found

227 that the U1snRNP binding to exon 5 donor site was enhanced when Calu-3 cells were treated with

228 CaNDY, as represented by the detection of the U1-70k major subunit in the pull-down products,

229 whereas phosphorylated SRSF6 not detected (OAS1SD pull-down products in Fig. 2c). Together, these

230 data indicate that SRSF6 plays a major role in the exon 5 donor site recognition, which is crucial for

231 OAS1 alternative splicing.

233 CaNDY induces splice shift in A-allele

234 Next, we investigated the consequence of CLK inhibition on alternative splicing of OAS1 mRNA with

235 A-allele. Calu-3 cells, lung adenocarcinoma cells homozygous for OAS1 A-allele (Fig. S1a), were

236 treated with $10 \mu \mathrm{M}$ CaNDY or DMSO and analyzed for $O A S 1$ splicing profiles, along with Daudi

237 cells, which are lymphoma cells homozygous for the G-allele (Fig. S1b). Consistent with previous

238 observations (Kjær et al., 2014; Noguchi et al., 2013), we found that the $p 46$ variant was dominantly 
239 expressed from OAS1 G-allele, while the $p 42$ variant was expressed from the A-allele (Fig. 2d).

240 Intriguingly, CLK inhibition induced splice-switching for $O A S 1$ with the A-allele, by suppressing $p 42$

241 splicing while promoting $p 44 a$ and $p 44 b$ variant production. This observation is consistent with the

242 model suggested by the RNA pull-down assay (Fig. 2c), where the exon 5 donor site is made available

243 for U1snRNP binding upon CLK inhibition (Fig. 2b). However, the $p 48$ variant expression was under

244 the detection limit in both A- and G-alleles (Fig. 2d). The splicing shift of OAS1 with A-allele upon

245 CLK inhibition was also evident in RNA-Seq analysis of Calu-3 cells, in which the $p 42$ variant was

246 suppressed by $51 \%$, and $p 44 a$ and $p 44 b$ were increased by $130 \%$ and $100 \%$, respectively; the $p 44 b$

247 variant was found to be a minor form of $O A S 1$, accounting for 1-4\% of $O A S 1$ transcripts (Fig. 2e, 2f).

248 Additionally, through the RNA-Seq analysis of Calu-3 cells with or without CaNDY treatment, we

249 identified differential expression of 98 genes, and splice alterations yielding protein-coding variants

250 for 63 genes; however, none of these events were associated with viral infection except for the OASI

251 splice alterations (Tables S2, S3, and Fig. S2).

253 SARS-CoV-2 resistance with splice shift

254 We examined the alternation of SARS-CoV-2 infection rate in Calu-3 cells, when the splicing isoform

255 was changed from $p 42$ to $p 44 a$ by manipulating the splicing phenomenon with a small chemical

256 compound. To sufficiently change the balance of the splicing isoforms, cells were pre-treated with

257 CaNDY for $24 \mathrm{~h}$. Viral titer was assayed 2 days after cells were exposed to the virus (Fig. 3a). The

258 virus titer decreased from $5.18 \times 10^{7}$ to $2.90 \times 10^{7}$ (fold change $\left.=0.56\right)$ in the cells treated with

259 CaNDY (Fig. 3b, Table S4). There are no reports on the enzyme activity or antiviral activity of the

260 p44a variant; however, based on our results, it can be assumed that the product encoded by $p 44 a$ has

261 stronger enzyme activity/antiviral ability than that encoded by the $p 42$ form. Furthermore, RNA

262 degradation products were measured to confirm whether this change was indeed due to the switching

263 of $O A S 1$ splicing isoforms. If enzyme activities of the product encoded by $O A S 1$ are elevated, elevated

264 activities of RNase L are expected to be observed. Activated RNase L can not only degrade the 
265 foreign RNA molecules, but also the endogenous ribosomal RNA within the host cells (Lin et al.,

266 2009). Thus, degradation of rRNA was investigated as a measure of RNase L activity. We confirmed

267 the appearance of a band that is expected to be a degradation product of rRNA in cells infected with

268 SARS-CoV-2 (Fig. 3c). We also confirmed that the concentration of decomposition products, sized

$2692,000-3,000$ nucleotides, increased slightly (fold change $=1.35$ ), with a decrease in the molecular

270 weight and concentration of the $28 \mathrm{~S}$ rRNA peak (fold change $=0.56$ ), upon CaNDY treatment (Fig.

$2713 c$, Fig. S3). It is known that degraded dsRNA activates the transcription of the interferon genes

272 through the OASL and RIG-I related pathways (Choi et al., 2015; Ibsen et al., 2015). We confirmed

273 that CaNDY treatment increases the transcription of interferon $\beta 1$ (IFNB1) during viral infection (Fig.

$2743 \mathrm{~d}, 3 \mathrm{e})$. This result was consistent with the previous reports showing IFNBI transactivation during

275 viral infections (Ford and Thanos, 2010; Schwanke et al., 2020).

276 These results indicate that CaNDY treatment enhances the infection-dependent RNase L pathway, and

277 the type I interferon pathway induced by the degraded RNAs, via a switching of OASI splicing

278 isoforms from $p 42$ to $p 44 a$ in Calu-3 cells (Fig. 3f).

280 Discussion

281 In this study, we show that the difference in the balance of $O A S 1$ splicing isoforms affects the

282 infectivity of SARS-CoV-2 in cells via modulation of the innate immune response. This result

283 strongly suggests that the OAS1 intervening sequence (IVS) 5-1 G> A SNP that alters the OAS1

284 splicing pattern is one of the important therapeutic targets for COVID-19. We recently reported on

285 CaNDY as a potential therapeutic drug for cystic fibrosis caused by a point mutation in the CFTR

286 gene (Shibata et al., 2020). In this study, we found that CaNDY treatment increased the yield of the

$287 p 44 a$ splice form (Fig. 2d, 2e, 2f) and made the Calu-3 cells more resistant to viral infection (Fig. 3b),

288 suggesting that CaNDY treatment could overcome A-allele-derived vulnerabilities to SARS-CoV-2

289 infections. 
290 The G-allele of SNP rs10774671 produces the $p 46$ splice variant, which possesses 56 amino acids at

291 the C-terminus, compared to the 18 completely different amino acids present in the $p 42$ splice variant

292 produced by the A-allele. This changes the localization of the OAS1 protein and its interacting

293 partners (Kjær et al., 2014), which may be linked to the differential 2'-5'-oligoadenylate synthesis

294 activities, RNase L activation, and interferon pathway activation. Several clinical trials that investigate

295 the efficacy of an intervention of the interferon $\beta$ pathway to affect the progression of COVID-19 have

296 been conducted successfully (Hung et al., 2020; Monk et al., 2021; Shalhoub, 2020). On the other

297 hand, inhibition of the interferon pathway is considered important to prevent cytokine storms in

298 patients with severe COVID-19 (Nile et al., 2020). Since the OAS1 IVS5-1 SNP can alter the response

299 intensity of the interferon $\beta$ pathway, stratification of COVID-19 patients depending on this SNP may

300 assist in choosing the best course of interferon treatment.

301 It is reported that protective SNPs at $O A S 1 / O A S 3 / O A S 2$ loci originated in the Neanderthals (Zeberg

302 and Pääbo, 2021; Zhou et al., 2021). Interestingly, the OAS1 IVS5-1 A-allele, which was confirmed to

303 be associated with the aggravation of COVID-19 in this study, was not found in the ancient human

304 genome, the sequenced Neanderthal genome, or the Denisovan genome (Mendez et al., 2013). This

305 suggests that the G to A mutation at the OAS1 IVS5-1 position occurred at a relatively modern age in

306 human history. The OAS1 IVS5-1 A-allele has expanded to become a major allele in the population,

307 especially in the Asian region (Fig. S4) (Marcus and Novembre, 2017). This suggests the possibility

308 that this variant has some positive effects on human survival, trading off the effect of weakening

309 resistance against viruses.

310 The results obtained in this study indicate that the OAS1 IVS5-1 SNP is involved in the exacerbation

311 of COVID-19 by eliciting changes in the OAS1 splicing isoform balance. Individuals with the A-allele

312 may have a higher risk for SARS-CoV-2 infection and its aggravation than those with the G-allele.

313 However, we successfully demonstrate a way to overcome these genetic risks by modulating the

314 splicing phenomenon. Splicing modulation decreased the virus infection rate 0.56 times in cell-based

315 assays (Fig. 3b). This theoretically implies that the peak number of SARS-CoV-2-infected individuals 
316 will be reduced by $42 \%$, if we can alter the $O A S 1$ splicing patterns for all the individuals carrying the

317 OAS1 IVS5-1 A-allele and boost the immunity of the entire population (simulation using the

318 Susceptible, Infected and Removed (SIR) model, see Methods for details). We hope that this approach

319 based on genome-based precision medicine with a splicing modulator will contribute to the

320 management of the COVID-19 pandemic.

\section{Acknowledgments}

323 We would like to thank Editage [http://www.editage.com] for editing and reviewing this manuscript

324 for English language.

\section{Conflict of Interest}

327 M.H. is a founder, shareholder, and member of the scientific advisory board of KinoPharma, Inc., and

328 BTB Drug Development Research Center Co., Ltd. All other authors have no competing interests.

\section{Author Contributions}

331 K.I. conducted bioinformatics analysis. M.A., M.D., and R.K. conducted biological experiments

332 relating cultured cells. Y.M., T.T., and T.N. conducted viral experiments. K.I., M.A., and M.H. wrote 333 the paper. 


\section{References}

337 Adam D. 2020. A guide to R — the pandemic's misunderstood metric. Nature 583:346-348.

$$
\text { doi:10.1038/d41586-020-02009-w }
$$

Blanco-Melo D, Nilsson-Payant BE, Liu W, Uhl S, Hoagland D, Møller R, Jordan TX, Oishi K, Panis M,

$$
\text { doi:10.1016/j.cell.2020.04.026 }
$$

Carey CM, Govande AA, Cooper JM, Hartley MK, Kranzusch PJ, Elde NC. 2019. Recurrent Loss-of-

Cartegni L, Wang J, Zhu Z, Zhang MQ, Krainer AR. 2003. ESEfinder: A web resource to identify exonic splicing enhancers. Nucleic Acids Res 31:3568-71. doi:10.1093/nar/gkg616

Choi UY, Kang J-S, Hwang YS, Kim Y-J. 2015. Oligoadenylate synthase-like (OASL) proteins: dual functions and associations with diseases. Exp Mol Med 47:e144. doi:10.1038/emm.2014.110

Di H, Elbahesh H, Brinton MA. 2020. Characteristics of Human OAS1 Isoform Proteins. Viruses 12.

Ford E, Thanos D. 2010. The transcriptional code of human IFN-beta gene expression. Biochim Biophys Acta 1799:328-36. doi:10.1016/j.bbagrm.2010.01.010

Hamano E, Hijikata M, Itoyama S, Quy T, Phi NC, Long HT, Ha LD, Ban V Van, Matsushita I, Yanai H, Kirikae F, Kirikae T, Kuratsuji T, Sasazuki T, Keicho N. 2005. Polymorphisms of interferon- 
nucleic acid polymorphisms of OAS1 and MxA genes: a case-control study. BMC Infect Dis 6:106. doi:10.1186/1471-2334-6-106

Hung IF-N, Lung K-C, Tso EY-K, Liu R, Chung TW-H, Chu M-Y, Ng Y-Y, Lo J, Chan J, Tam AR, Shum H-P, Chan V, Wu AK-L, Sin K-M, Leung W-S, Law W-L, Lung DC, Sin S, Yeung P, Yip CC-Y, Zhang RR, Fung AY-F, Yan EY-W, Leung K-H, Ip JD, Chu AW-H, Chan Wan-Mui, Ng AC-K, Lee

Ibsen MS, Gad HH, Andersen LL, Hornung V, Julkunen I, Sarkar SN, Hartmann R. 2015. Structural and functional analysis reveals that human OASL binds dsRNA to enhance RIG-I signaling. Nucleic Acids Res 43:5236-48. doi:10.1093/nar/gkv389

Kjær KH, Pahus J, Hansen MF, Poulsen JB, Christensen EI, Justesen J, Martensen PM. 2014.

Lin R-J, Yu H-P, Chang B-L, Tang W-C, Liao C-L, Lin Y-L. 2009. Distinct antiviral roles for human 2',5'oligoadenylate synthetase family members against dengue virus infection. J Immunol 183:8035-43.

Marcus JH, Novembre J. 2017. Visualizing the geography of genetic variants. Bioinformatics 33:594-595. doi:10.1093/bioinformatics/btw643

Mendez FL, Watkins JC, Hammer MF. 2013. Neandertal origin of genetic variation at the cluster of OAS immunity genes. Mol Biol Evol 30:798-801. doi:10.1093/molbev/mst004 
SARS-CoV-2 infection: a randomised, double-blind, placebo-controlled, phase 2 trial. Lancet Respir Med 9:196-206. doi:10.1016/S2213-2600(20)30511-7

391 Nile SH, Nile A, Qiu J, Li L, Jia X, Kai G. 2020. COVID-19: Pathogenesis, cytokine storm and therapeutic potential of interferons. Cytokine Growth Factor Rev 53:66-70. doi:10.1016/j.cytogfr.2020.05.002

Noguchi S, Hamano E, Matsushita I, Hijikata M, Ito H, Nagase T, Keicho N. 2013. Differential effects of a

Pairo-Castineira E, Clohisey S, Klaric L, Bretherick AD, Rawlik K, Pasko D, Walker S, Parkinson N, Fourman MH, Russell CD, Furniss J, Richmond A, Gountouna E, Wrobel N, Harrison D, Wang B, Wu Y, Meynert A, Griffiths F, Oosthuyzen W, Kousathanas A, Moutsianas L, Yang Z, Zhai R, Zheng C, Grimes G, Beale R, Millar J, Shih B, Keating S, Zechner M, Haley C, Porteous DJ, Hayward C, Yang J, Knight J, Summers C, Shankar-Hari M, Klenerman P, Turtle L, Ho A, Moore SC, Hinds C, Horby P, Nichol A, Maslove D, Ling L, McAuley D, Montgomery H, Walsh T, Pereira AC, Renieri A, GenOMICC Investigators, ISARIC4C Investigators, COVID-19 Human Genetics Initiative, 23andMe Investigators, BRACOVID Investigators, Gen-COVID Investigators, Shen X, Ponting CP, Fawkes A, Tenesa A, Caulfield M, Scott R, Rowan K, Murphy L, Openshaw PJM, Semple MG, Law A, Vitart V, Wilson JF, Baillie JK. 2021. Genetic mechanisms of critical illness in COVID-19. Nature 591:92-98. doi:10.1038/s41586-020-03065-y

Sadler AJ, Williams BRG. 2008. Interferon-inducible antiviral effectors. Nat Rev Immunol 8:559-68. doi:10.1038/nri2314

Sakuma M, Iida K, Hagiwara M. 2015. Deciphering targeting rules of splicing modulator compounds: case of TG003. BMC Mol Biol 16:16. doi:10.1186/s12867-015-0044-6

Schlee M, Hartmann G. 2016. Discriminating self from non-self in nucleic acid sensing. Nat Rev Immunol 16:566-80. doi:10.1038/nri.2016.78

Schwanke H, Stempel M, Brinkmann MM. 2020. Of Keeping and Tipping the Balance: Host Regulation and Viral Modulation of IRF3-Dependent IFNB1 Expression. Viruses 12. doi:10.3390/v12070733 
415 Shalhoub S. 2020. Interferon beta-1b for COVID-19. Lancet (London, England) 395:1670-1671. doi:10.1016/S0140-6736(20)31101-6

417 Shibata S, Ajiro M, Hagiwara M. 2020. Mechanism-Based Personalized Medicine for Cystic Fibrosis by Suppressing Pseudo Exon Inclusion. Cell Chem Biol 27:1472-1482.e6. doi:10.1016/j.chembiol.2020.08.013

420 Soetaert K, Petzoldt T, Setzer RW. 2010. Solving Differential Equations in R : Package deSolve. J Stat Softw 33. doi:10.18637/jss.v033.i09

422 The COVID-19 Host Genetics Initiative. 2021. Mapping the human genetic architecture of COVID-19 by 423 worldwide meta-analysis. medRxiv 2021.03.10.21252820. doi:10.1101/2021.03.10.21252820

424 Zeberg H, Pääbo S. 2021. A genomic region associated with protection against severe COVID-19 is 425 inherited from Neandertals. Proc Natl Acad Sci U S A 118. doi:10.1073/pnas.2026309118

426 Zhou S, Butler-Laporte G, Nakanishi T, Morrison DR, Afilalo J, Afilalo M, Laurent L, Pietzner M,

427 Kerrison N, Zhao K, Brunet-Ratnasingham E, Henry D, Kimchi N, Afrasiabi Z, Rezk N, Bouab M,

428 Petitjean L, Guzman C, Xue X, Tselios C, Vulesevic B, Adeleye O, Abdullah T, Almamlouk N, Chen

429 Y, Chassé M, Durand M, Paterson C, Normark J, Frithiof R, Lipcsey M, Hultström M, Greenwood

430 CMT, Zeberg H, Langenberg C, Thysell E, Pollak M, Mooser V, Forgetta V, Kaufmann DE,

431 Richards JB. 2021. A Neanderthal OAS1 isoform protects individuals of European ancestry against

432 COVID-19 susceptibility and severity. Nat Med. doi:10.1038/s41591-021-01281-1 
a Very severe respiratory confirmed COVID-19

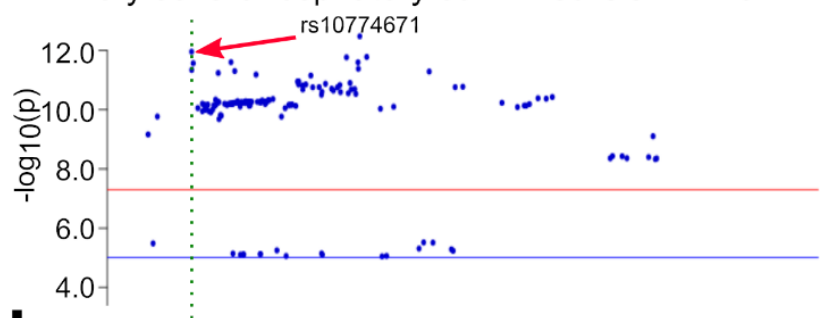

b Hospitalized COVID-19 patients

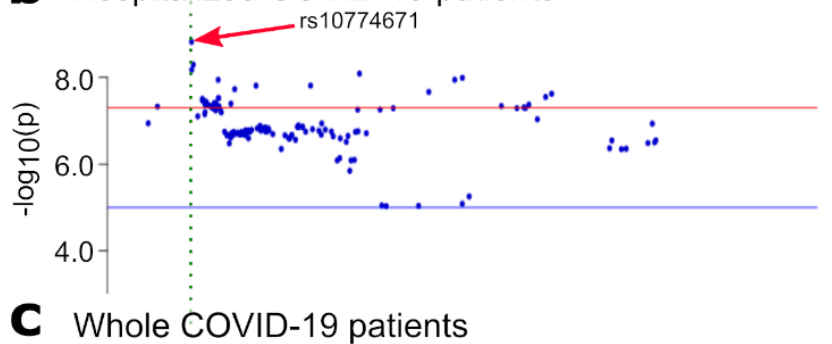

C Whole COVID-19 patients

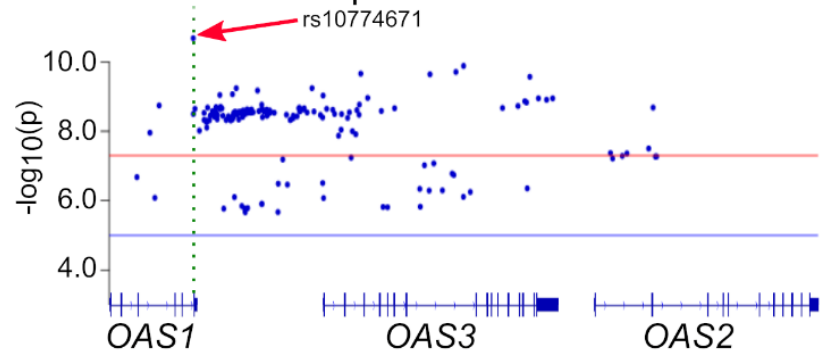

Figure 1. OAS1-3 loci have COVID-19 associated SNPs and respond to SARS-CoV-2

439 Positions and GWAS p-values for SNPs on $O A S 1, O A S 3$, and $O A S 2$ gene loci. GWAS results for

440 confirmed COVID-19 cases with very severe respiratory conditions (a), hospitalized COVID-19

441 patients (b), and all COVID-19 patients (c), reported by COVID-19 Host Genome Initiative groups,

442 are shown (Reported by COVID-19 Host Genome Initiative groups). Red and blue horizontal lines

443 show the p-value positions of 5E-8 and 1E-5, respectively. The position of rs10774671 SNP is shown

444 with a green dotted line and red arrows. 
a
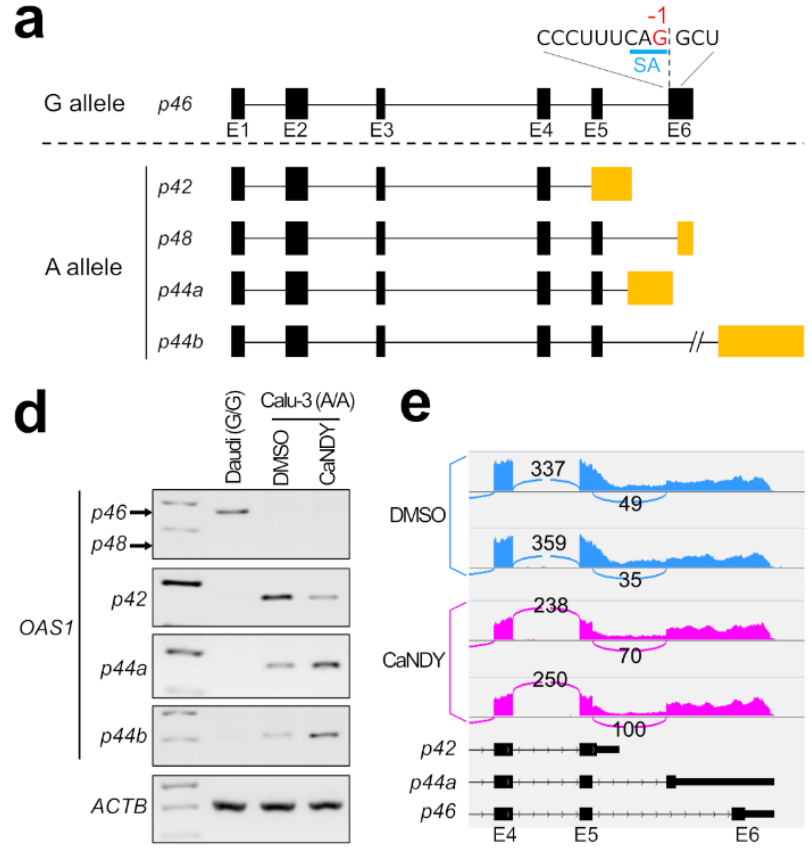

e

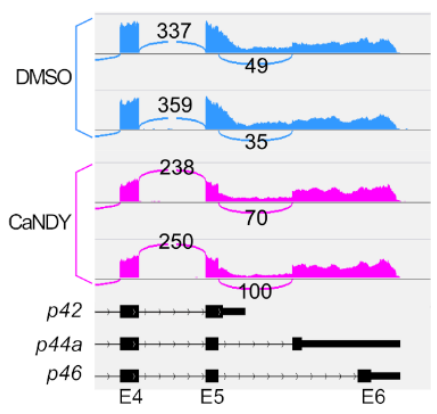

b

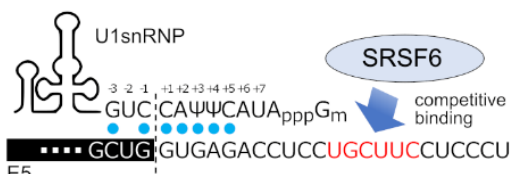

splice junction
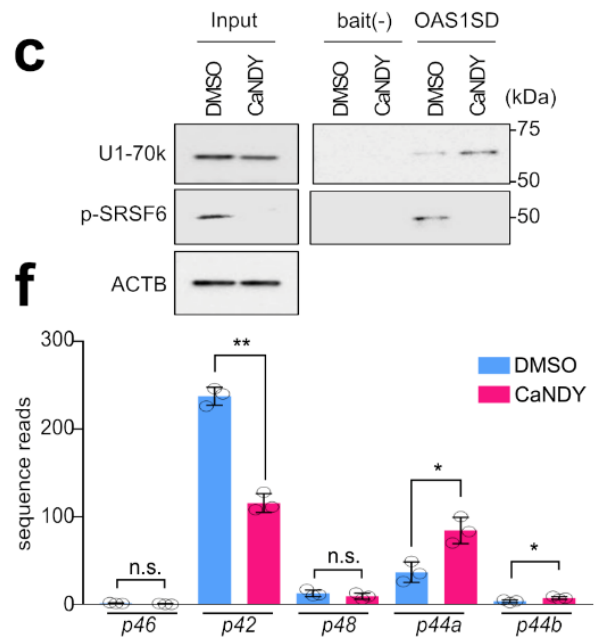

Figure 2. CLK inhibitor CaNDY induces splice-switching in OAS1 rs10774671 A allele.

450 a, Allele-dependent $O A S 1$ alternative splicing. Pre-mRNA of $O A S 1$, with the rs $10774671 \mathrm{G}$-allele at -

4511 position of exon 6 splice acceptor (SA), dominantly produces the $p 46$ variant, whereas the A-allele

452 leads to alternative splicing to produce the $p 42, p 48, p 44 a$, and $p 44 b$ variants with differences in the

453 last exon (yellow boxes). b, The SRSF6 binding motif (red letters) present in close vicinity of the U1

454 binding site of $O A S 1$ donor site for $p 44 a / p 44 b / p 48$ splicing. Blue dots indicate U1snRNA pairing. c,

455 Western blot of U1-70k and phosphorylated SRSF6 (p-SRSF6) for RNA pull-down products of OAS1

456 exon 5 splice donor (SD) sequence in Calu-3 cells treated with $0.1 \%$ DMSO or $10 \mu \mathrm{M}$ CaNDY. Input,

457 input samples; bait (-), negative control products without the bait RNA oligo. ACTB served as the

458 loading control. d, RT-PCR for OAS1 alternative splicing profile in Daudi (G/G allele) and Calu-3

459 (A/A allele) cells treated with $0.1 \%$ DMSO or $10 \mu \mathrm{M}$ CaNDY for $24 \mathrm{~h}$. ACTB served as a loading

460 control. e, RNA-Seq results indicated by a Sashimi plot for a region covering $p 42, p 44 a$, and $p 46$. f, 
461 Numbers of splice-junction reads for each $O A S 1$ splice variants. Dots, read number of repeats; error

462 bars, \pm SD. n.s., $\mathrm{p} \geq 0.05 ; *, \mathrm{p}<0.05 ; * *, \mathrm{p}<0.01$ by Student's t-test.

463

464 Figure 2-source data 1 (Separated File)

465 Original Western blot files for Figure 2C.

466

467 Figure 2-source data 2 (Separated File)

468 Original gel electrophoresis files for Figure 2D. 


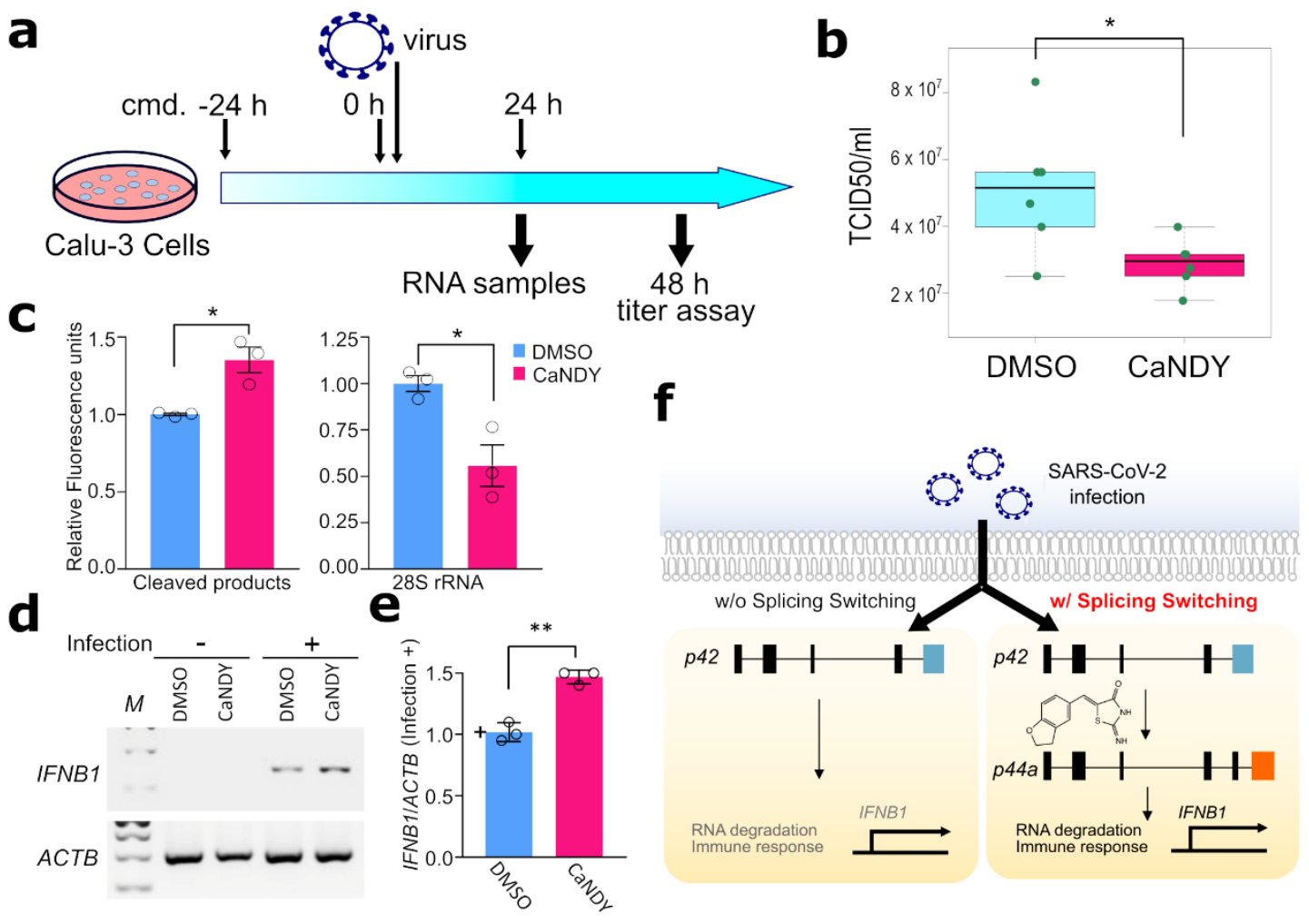

472 Figure 3. Pre-treatment with CaNDY confers Calu-3 cells with resistance against SARS-CoV-2

473 infection via activation of the $R$ Nase $\mathbf{L}$ and interferon pathways. a, A scheme for the pre-treatment

474 of CaNDY, virus titer assay. b, Box plots showing logarithm translated virus titers. The shown values

475 are corrected for batch effects (See methods and Table S4). c, Bar-plot for cleaved RNA products and

476 28S rRNA measured with the BioAnalyzer system for the RNA samples from Calu-3 cells, before and

477 after the viral infection for $24 \mathrm{~h}$, with and without CaNDY treatments. Error bars, $\pm \mathrm{SD} ; *, \mathrm{p}<0.05$; by

478 Student's t-test. d, RT-PCR results for IFNBI mRNA. e, Bar-plot for quantified IFNB1/ACTB in the

479 infected cells based on RT-PCR results. Error bars, $\pm \mathrm{SD} ; * *, \mathrm{p}<0.01$; by Student's t-test. f, A

480 summary of the current study.

482 Figure 3-source data 1 (Separated File)

483 Original BioAnalyzer system result file for Figure 3C. 
bioRxiv preprint doi: https://doi.org/10.1101/2021.08.23.457314; this version posted August 23, 2021. The copyright holder for this preprint (which was not certified by peer review) is the author/funder, who has granted bioRxiv a license to display the preprint in perpetuity. It is made available under aCC-BY 4.0 International license.

484

$485 \quad$ Figure 3-source data 2 (Separated File)

486 Original gel electrophoresis files for Figure 3D.

487 
488 Table 1. SNPs on $O A S 1$ locus associated with very severe respiratory confirmed COVID-19 (p $<5$ x

$\left.48910^{-8}\right)$.

490

\begin{tabular}{|c|c|c|c|c|c|c|c|}
\hline Chr & Pos & ID & p-value ${ }^{*}$ & $\begin{array}{l}\log \\
\text { (Odds } \\
\text { Ratio) } \\
*\end{array}$ & Ref & Alt & $\begin{array}{l}\text { Location to OAS1 transcript } \\
\text { variant } 1(\mathrm{p} 46)\end{array}$ \\
\hline 12 & $112,912,991$ & rs2057778 & $6.86 \times 10^{-10}$ & 0.17 & $\mathrm{G}$ & $\mathrm{T}$ & $\begin{array}{l}\text { Intron } 3 \text { (1756th base of } \\
\text { 5273nt.) }\end{array}$ \\
\hline 12 & $112,914,354$ & rs4767023 & $1.70 \times 10^{-10}$ & 0.17 & $\mathrm{~T}$ & $\mathrm{C}$ & $\begin{array}{l}\text { Intron } 3 \text { (3119th base of } \\
\text { 5273nt.) }\end{array}$ \\
\hline 12 & $112,919,388$ & rs10774671 & $1.10 \times 10^{-12}$ & 0.18 & $G$ & A & $\begin{array}{l}\text { Intron } 5 \text { (1688th base of } \\
1688 \text { nt.) }\end{array}$ \\
\hline 12 & $112,919,404$ & rs1131476 & $4.54 \times 10^{-12}$ & 0.18 & $\mathrm{G}$ & $\bar{A}$ & $\begin{array}{l}\text { Exon } 6 \text { (16th base of 515nt., } \\
\text { CDS, Ala>Thr) }\end{array}$ \\
\hline 12 & $112,919,637$ & rs2660 & $2.68 \times 10^{-12}$ & 0.19 & $\mathrm{G}$ & $\mathrm{A}$ & $\begin{array}{l}\text { Exon } 6 \text { (249th base of 515nt., } \\
\text { 3' UTR) }\end{array}$ \\
\hline
\end{tabular}

491 


\section{Supplementary Information for}

Switching of OAS1 splicing isoforms mitigates SARS-CoV-2 infection

Kei Iida, Masahiko Ajiro, Yukiko Muramoto, Toru Takenaga, Masatsugu Denawa, Ryo

Kurosawa, Takeshi Noda, Masatoshi Hagiwara

Kei Iida, Masatoshi Hagiwara

Email: iida.kei.3r@kyoto-u.ac.jp, hagiwara.masatoshi.8c@kyoto-u.ac.jp

\section{This Supplementary Information includes:}

Figures $\mathrm{S} 1$ to $\mathrm{S} 4$

Table S1

Legends for Tables S2, S3

Table S4

SI References

Other supplementary materials for this manuscript include the following:

Tables S2 to S3 
a Calu-3

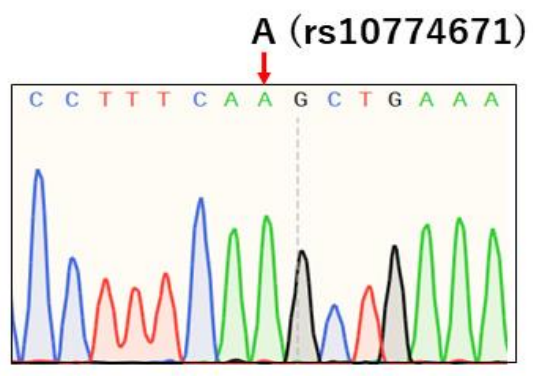

b Daudi

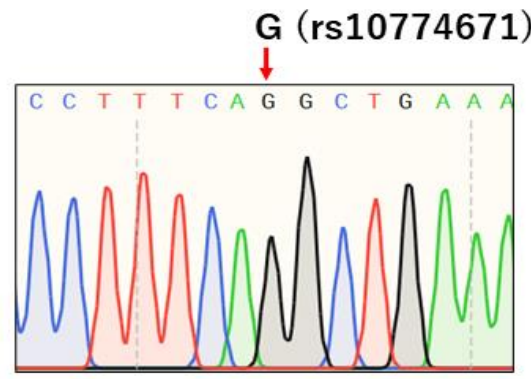

Fig. S1. Genotyping of rs10774671 SNP. Results of the SNP genotyping for Calu-3 cells (a) and Daudi cells (b). 
a Up-regulated genes (98 genes)

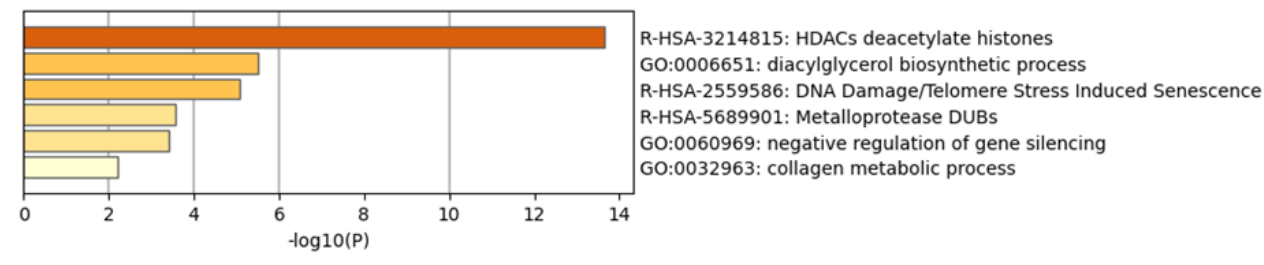

b Genes with altered splicing events yielding protein-codingvariants (63 genes)

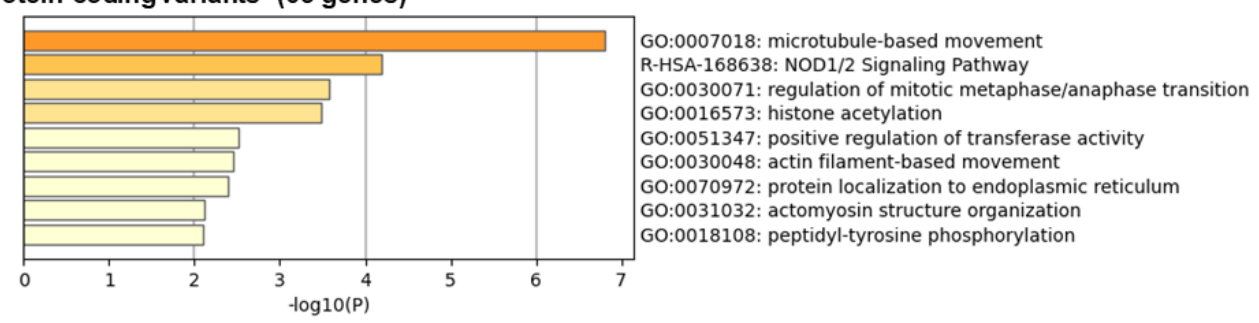

Fig. S2. Pathway analysis for CaNDY-affected genes. Pathway analyses for 98 differentially up-regulated genes (a) and 63 differentially alternative spliced genes (b) in CaNDY-treated Calu-3 cells are shown. For the differentially alternative spliced genes, we used the splicing events that promote productive forms (see Methods for details). These analyses were performed with the Metascape web server (Tripathi et al., 2015) 
a

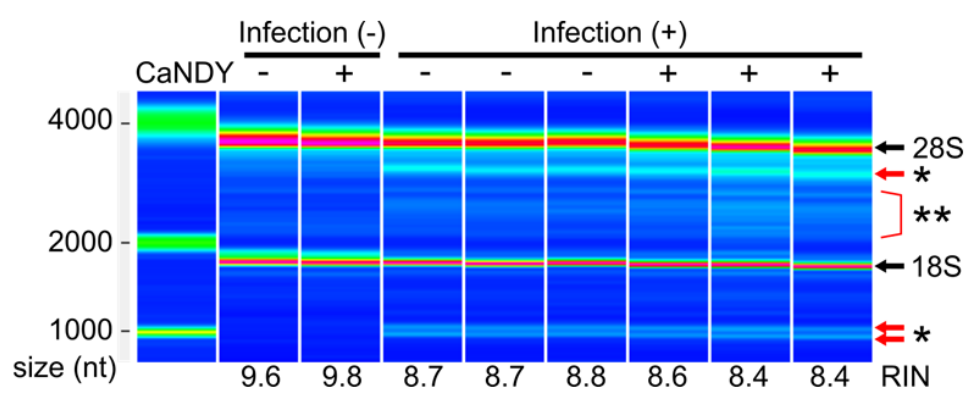

b

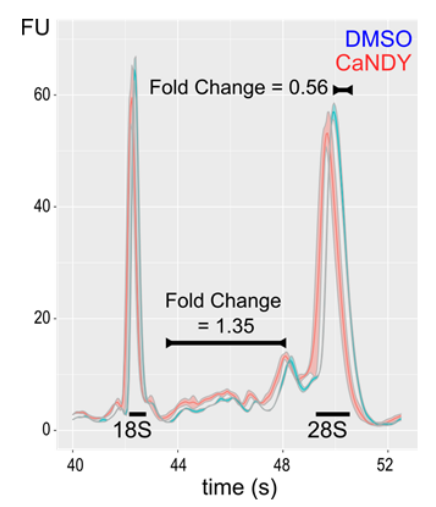

Fig. S3. RNA degradation in SARS-CoV-2-infected cells. (a) A gel image was obtained based on the results from the Bioanalyzer. (b) Line graphs for fluorescence units according to migration times. Solid lines show the mean values and ribbons show the range of standard errors. 


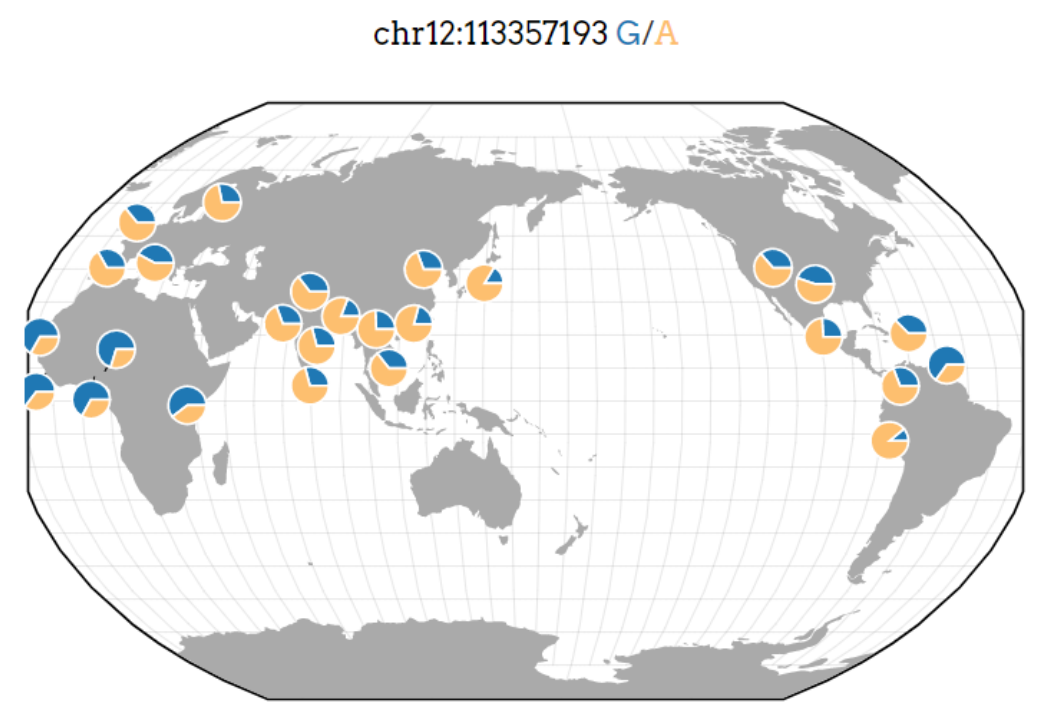

Fig. S4. Allele frequency of the rs10774671 SNP. A world map showing the relationship between the location and allele frequency of the SNP. The position in the hg19 genome is shown. The map was obtained from the Geography of Genetic Variants (GGV) browser (Marcus and Novembre, 2017). 
bioRxiv preprint doi: https://doi.org/10.1101/2021.08.23.457314; this version posted August 23, 2021. The copyright holder for this preprint (which was not certified by peer review) is the author/funder, who has granted bioRxiv a license to display the preprint in perpetuity. It is made available under aCC-BY 4.0 International license.

Table S1. List of primers used in this study

\begin{tabular}{|c|c|c|c|c|}
\hline Primer name & orientation & Target gene & Target isoform & Sequence $\left(5^{\prime}\right.$-to- $\left.3^{\prime}\right)$ \\
\hline oAM13 & Forward & $A C T B$ & & CCAACCGCGAGAAGATGACC \\
\hline oAM14 & Reverse & ACTB & & AGCTTCTCCTTAATGTCACG \\
\hline oAM674 & Forward & OAS1 & OAS1 p42, OAS1 p44a, OAS1 p44b, OAS1 p46, OAS1 p48 & GGTTGGAGGCAGCTGGCAC \\
\hline oAM675 & Reverse & OAS1 & OAS1 p42 & CCAGCTATATGTCTCAAGCT \\
\hline oAM676 & Reverse & OAS1 & OAS1 p46, OAS1 p48 & CTGGCATTCAGAGGATGGTG \\
\hline oAM677 & Reverse & OAS1 & OAS1 p44b & CTGCСTCССTAAGCAACCTG \\
\hline oAM678 & Reverse & OAS1 & OAS1 p44a & CСATTTCCACCACTTGTTAG \\
\hline oAM700 & Forward & IFNB1 & & CAGTGTCAGAAGCTCCTGTG \\
\hline oAM701 & Reverse & IFNB1 & & ATCTGATGATAGACATTAGC \\
\hline
\end{tabular}


bioRxiv preprint doi: https://doi.org/10.1101/2021.08.23.457314; this version posted August 23, 2021. The copyright holder for this preprint (which was not certified by peer review) is the author/funder, who has granted bioRxiv a license to display the preprint in perpetuity. It is made available under aCC-BY 4.0 International license.

Table S2. Up-regulated Genes in CaNDY-treated Calu-3 cells (Separate File)

Table S3. Altered splicing events yielding protein-coding variants in CaNDY-treated Calu-3 cells (Separate File) 
bioRxiv preprint doi: https://doi.org/10.1101/2021.08.23.457314; this version posted August 23,2021 . The copyright holder for this preprint (which was not certified by peer review) is the author/funder, who has granted bioRxiv a license to display the preprint in perpetuity. It is made available under aCC-BY 4.0 International license.

Table S4. Raw and Batch-effect corrected results of the virus titer assay

\begin{tabular}{|c|c|c|c|c|}
\hline & Treatment & No. & $\log _{10}\left(\mathrm{TCID}_{50} / \mathrm{ml}\right)$ & Batch-effect corrected \\
\hline \multirow{6}{*}{$1^{\text {st }}$ batch } & \multirow{3}{*}{ CaNDY $0 \mu \mathrm{M}$} & 1 & 7.75 & - \\
\hline & & 2 & 7.67 & - \\
\hline & & 3 & 7.75 & - \\
\hline & \multirow{3}{*}{ CaNDY $10 \mu \mathrm{M}$} & 1 & 7.50 & - \\
\hline & & 2 & 7.50 & - \\
\hline & & 3 & 7.25 & - \\
\hline \multirow{6}{*}{$2^{\text {nd }}$ batch } & \multirow{3}{*}{ CaNDY $0 \mu \mathrm{M}$} & 1 & 6.75 & 7.60 \\
\hline & & 2 & 7.25 & 7.92 \\
\hline & & 3 & 6.50 & 7.44 \\
\hline & \multirow{3}{*}{ CaNDY $10 \mu \mathrm{M}$} & 1 & 6.75 & 7.60 \\
\hline & & 2 & 6.50 & 7.44 \\
\hline & & 3 & 6.45 & 7.41 \\
\hline
\end{tabular}




\section{SI References}

Marcus JH, Novembre J. 2017. Visualizing the geography of genetic variants.

Bioinformatics 33:594-595. doi:10.1093/bioinformatics/btw643

Tripathi S, Pohl MO, Zhou Y, Rodriguez-Frandsen A, Wang G, Stein DA, Moulton HM, DeJesus P, Che J, Mulder LCF, Yángüez E, Andenmatten D, Pache L, Manicassamy B, Albrecht RA, Gonzalez MG, Nguyen Q, Brass A, Elledge S, White M, Shapira S, Hacohen N, Karlas A, Meyer TF, Shales M, Gatorano A, Johnson JR, Jang G, Johnson T, Verschueren E, Sanders D, Krogan N, Shaw M, König R, Stertz S, García-Sastre A, Chanda SK. 2015. Meta- and Orthogonal Integration of Influenza "OMICs" Data Defines a Role for UBR4 in Virus Budding. Cell Host Microbe 18:723-35. doi:10.1016/j.chom.2015.11.002 